Network Working Group

Request for Comments: 1150

FYI : 1
G. Malkin

Proteon

J. Reynolds

ISI

March 1990

F.Y.I. on F.Y.I.

Introduction to the F.Y.I. Notes

Status of this Memo

This RFC is the first in a new sub-series of RFCs called FYIs (For Your Information). This memo provides information for the Internet community. It does not specify any standard. Distribution of this memo is unlimited.

1. Introduction

The FYI series of notes is designed to provide Internet users with a central repository of information about any topics which relate to the Internet. FYIs topics may range from historical memos on "Why it was was done this way" to answers to commonly asked operational questions.

The FYIs are intended for a wide audience. Some FYIs will cater to beginners, while others will discuss more advanced topics. An FYI may be submitted by anyone who has something to contribute and has the time to do so.

2. Why RFCS

There are several reasons why the FYIs are part of the larger RFC series of notes. The formost reason is that the distribution mechanisms for RFCs are tried and true. Anyone who can get an RFC, can automatically get an FYI. More importantly, anyone who knows of the RFC series, can easily find out about the FYIs.

Another reason for making FYIs part of the RFC series is that the maintainance mechanisms for RFCs are already in place and funded. It makes sense to maintain similar documents is a similar way. After all, there have been informational RFCs before.

Finally, the name RFC has come to carry a meaning with it. There is credibility associated memos carrying the RFC label. FYIs should share that respect. 


\section{Format Rules}

Since the FYIs are a part of the RFC series, they must conform to RFC-1111 (Request for Comments on Request for Comments: Instructions to RFC Authors) with respect to format. Ideally, they should be submitted in ASCII format, as described by section 2a, of RFC-1111.

4. Status statement

Each RFC must include on its first page the "Status of this Memo" section which contains a paragraph describing the intention of the RFC. This section is meant to convey the status granted by the RFC Editor and the Internet Activities Board (IAB). There are several reasons for publishing a memo as an $\mathrm{RFC}$, for example, to make available some information for interested people, or to begin or continue a discussion of an interesting idea, or to make available the specification of a protocol.

For example:

This RFC is the first in a new sub-series of RFCs called FYIs (For Your Information). This memo provides information for the Internet community. It does not specify any standard. Distribution of this memo is unlimited.

5. Distribution statement

Each FYI is to also include a "distribution statement". As the purpose of the FYI series is to disseminate information, there is no reason for the distribution to be anything other than "unlimited".

Typically, the distribution statement will simply be the sentence "Distribution of this memo is unlimited." appended to the "Status of this Memo" section.

6. Security Considerations

All FYIs must contain a section that discusses the security considerations of the procedures that are the main topic of the RFC.

7. Author's Address

Each FYI must have at the very end a section giving the author's address, including the name and postal address, the telephone number, and the Internet email address. 


\section{Relation to other FYIs}

Sometimes an FYI adds information on a topic discussed in a previous FYI or completely replaces an earlier FYI. There are two terms used for these cases respectively, UPDATES and OBSOLETES. A document that obsoletes an earlier document can stand on its own. A document that merely updates an earlier document cannot stand on its own; it is something that must be added to or inserted into the existing document, and has limited usefulness independently.

\section{UPDATES}

To be used as a reference from a new item that cannot be used alone (i.e., one that supplements a previous document), to refer to the previous document. The newer publication is a part that will supplement or be added on to the existing document; e.g., an addendum, or separate, extra information that is to be added to the original document.

\section{OBSOLETES}

To be used to refer to an earlier document that is replaced by this document. This document contains either revised information, or else all of the same information plus some new information, however extensive or brief that new information is; i.e., this document can be used alone, without reference to the older document.

OBSOLETED-BY

To be used to refer to the newer document that replaces the older document.

UPDATED-BY

To be used to refer to the newer document that adds information to the existing, still useful, document.

9. The FYI Editors

All FYIs are submitted to the IETF User Services Working Group for review prior to their submission to the RFC Editor.

Submissions may be made to: 


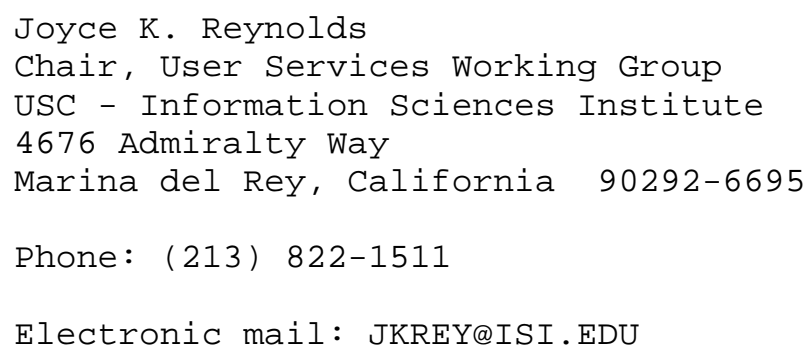

10. The FYI Announcement List

New FYIs are announced to the RFC distribution list maintained by the SRI Network Information Center (NIC). Contact the SRI-NIC to be added or deleted from this mailing list by sending an email message to RFC-REQUEST@NIC.DDN.MIL.

\section{Obtaining FYIs}

FYIs can be obtained via FTP from NIC.DDN.MIL, with the pathname FYI:mm.TXT, or RFC:RFCnnnn.TXT (where "mm" refers to the number of the FYI and "nnnn" refers to the number of the RFC). Login with FTP, username ANONYMOUS and password GUEST. The NIC also provides an automatic mail service for those sites which cannot use FTP. Address the request to SERVICE@NIC.DDN.MIL and in the subject field of the message indicate the FYI or RFC number, as in "Subject: FYI mm" or "Subject: RFC nnnn".

Security Considerations

Security issues are not discussed in this memo.

Authors' Addresses

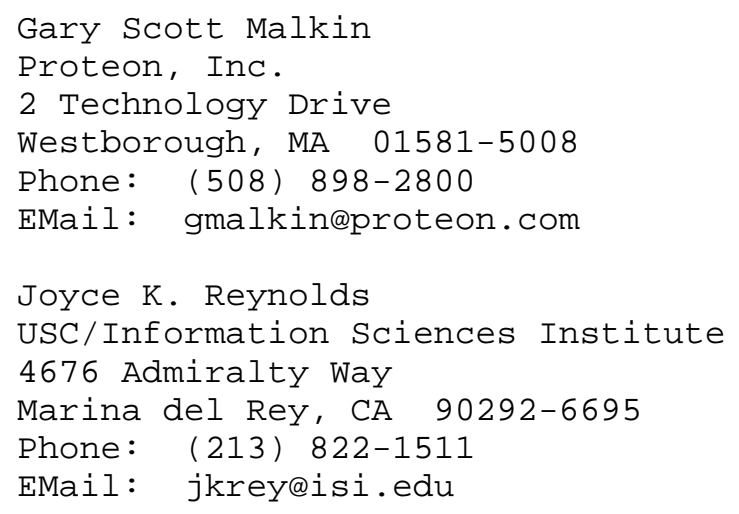

